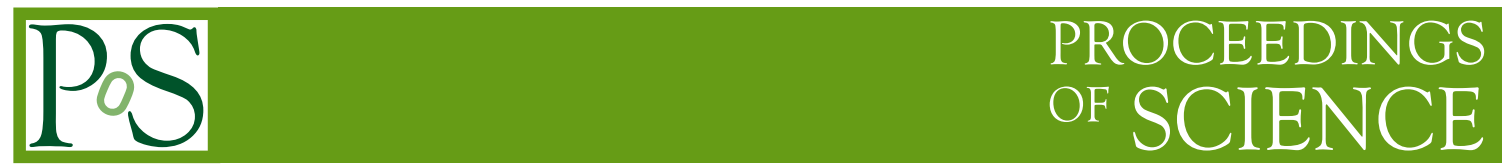

\title{
Supersonic gas Jet target for nuclear physics experiments
}

\author{
Francisco Favela ${ }^{* i}$ \\ IFUNAM \\ E-mail: ffavela@gmail.com
}

\section{Efraín Chávez, Eduardo Andrade, Oscar de Lucio, M. E. Ortíz}

Instituto de Física, Universidad Nacional Autónoma de México Ciudad Universitaria, 04510

Coyoacán D. F. México.

A supersonic gas jet target has been designed and constructed at the Institute of Physics in Mexico City. Details on its design and construction and on its characterization using Air, Nitrogen and Argon will be given.

X Latin American Symposium on Nuclear Physics and Applications (X LASNPA),

1-6 December 2013

Montevideo, Uruguay

${ }^{*}$ Speaker.

${ }^{\dagger}$ Thanks to CONACYT and DGAPAUNAM for helping to make this possible. 


\section{Introduction}

The "Carlos Graef Fernández Building" is home to the 5.5 MV CN-Van de Graaff accelerator at the Instituto de Física of the Universidad Nacional Autónoma de México (IFUNAM). The laboratory has recently been upgraded to extend the number of beam lines from 1 to 7 . One of these beam lines has been devoted to the development of the supersonic gas jet target presented here. A supersonic jet target is essentially a well-defined high density region of a given gas within a chamber that can be coupled to a high vacuum system (an accelerator beam line) using a complex differential pumping system.

It's able to withstand all the beam current any accelerator could give without changing its properties, which makes it ideal for low count rate experiments. Similar systems have been successfully developed for nuclear physics experiments in the past [1, 2, 3, 4]. Our modular design allows for simple upgrades in the future.

The relative loss in density compared to a solid target can be largely compensated by the increased beam intensity that the target can stand, resulting in a net increase the counting rate on any experiment.

\section{Experimental Methods}

In figure 1 to the left, a top view sketch of the laboratory is shown. The beam from the vertical accelerator is deflected by a 90 degree analyzing magnet, shown at the left of the image. The beam passes through a magnetic quadrupole and a beam diagnostics unit until it reaches the switching magnet, where the beam can be directed to the line of the jet (the top one in the figure).
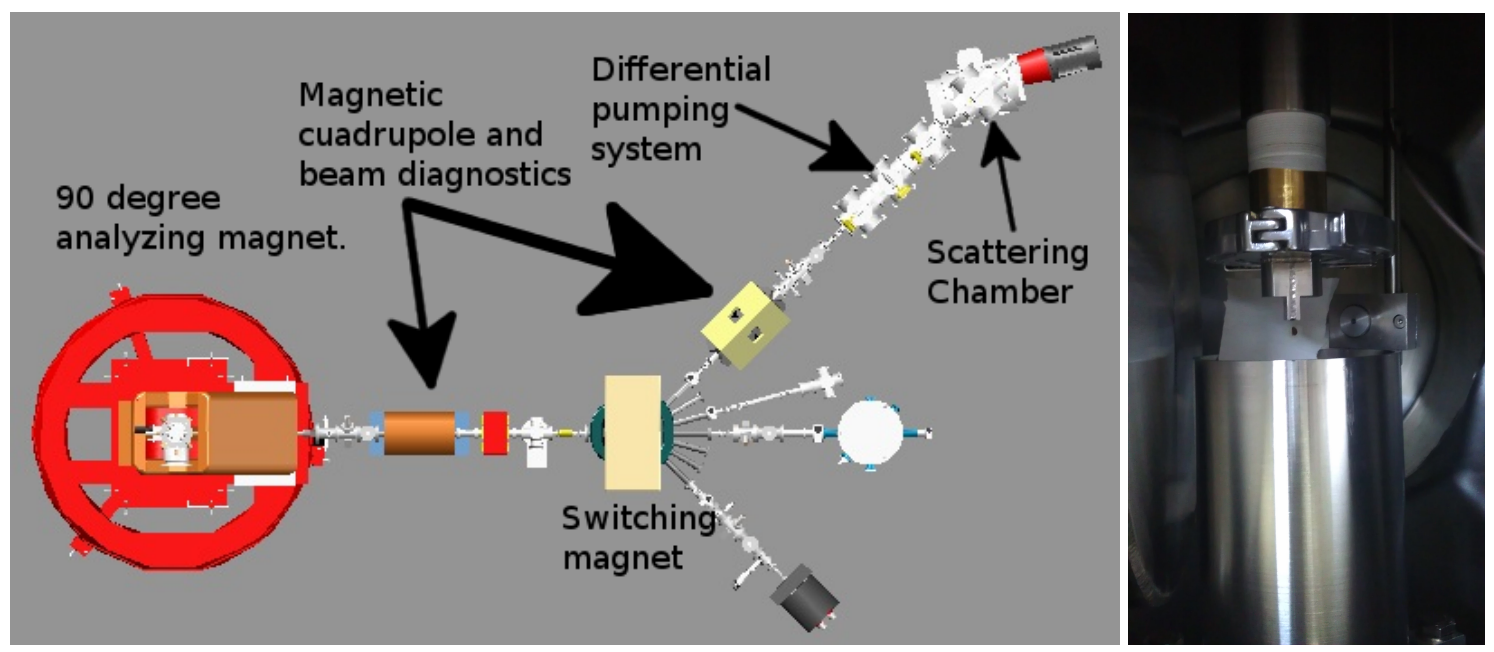

Figure 1: Sketch of the laboratory (left) and a picture of the nozzle inside the scattering chamber (right).

Right after the valve isolating our beam line from the switching magnet there is a second diagnostics unit. Coupled operation of these two beam diagnostics units provide continuous information about the position and intensity of the beam delivered to our beam line.

The differential pumping system is a sequence of chambers with independent high vacuum systems. In between each chamber there is a $5 \mathrm{~mm}$ circular aperture. This aperture is designed to 
be big enough to let most of the beam to go through and small enough to limit the gas flow (or throughput) from one chamber to the next.

This allows us to connect a high pressure region, the scattering chamber where the jet is located, with the high vacuum system of the accelerator (several orders of magnitude lower).

Past the scattering chamber, one last Faraday cup was used mostly for alignment optimization by in measuring the amount of beam that reached the end of the beam line. Typically $60 \%$ of the beam delivered to our beam line was transmitted through all chambers and apertures to the end.

The inside of the scattering chamber can be seen in the right side of figure 1. A rectangular nozzle was used, most of the gas gets caught by a central pipe that is connected to a high speed mechanical pump. The spilled gas is collected by a high speed Roots pumping system.

At the back of the picture, one of the EBS (elastic backscattering spectroscopy) detectors can be seen, placed at a 150 degrees with respect the front of the beam. As part of the alignment, a piece of paper was placed right before the nozzle, in order for the beam to burn it. As expected the beam burned the paper just below the nozzle, as seen in 1 right.

\section{Results and conclusions}

Figure 2 shows a series of images taken from one of the used nozzles. A special technique (Schlieren [5, 6]) was used in order to visualize the density differences in transparent media.

The used gas was air, the experiment was at a fluids laboratory, before the gas jet beamline was set.
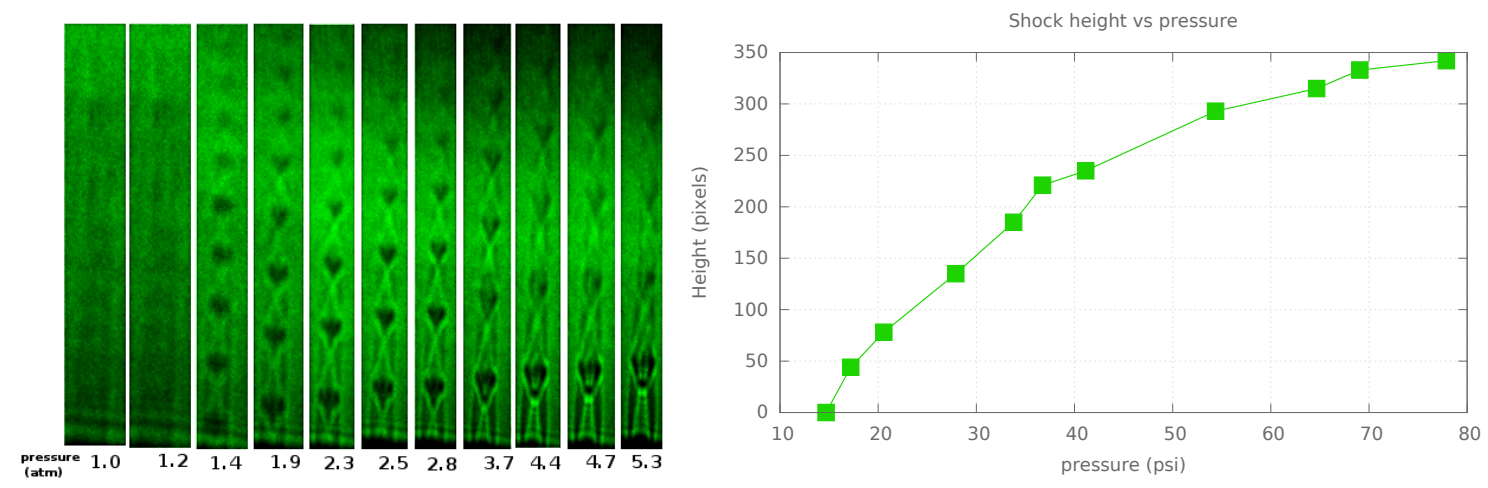

Figure 2: Schlieren images from the supersonic jet flow of air (left). Height of the first shock zone ("tonge") at different pressures right.

In this measurements the pressure at the exit of the nozzle is $1 \mathrm{Bar}(\approx 1 \mathrm{~atm})$. Once the ratio of the pressure at the entrance of the nozzle to that at the exit achieves a critical condition (around 2.5) the flow becomes supersonic. The first shock region or "tongue", is the region to be used as a target. We can conclude that height of the tongue increases non-linearly with the input pressure. The behavior can also be seen in the plot on the right side of figure 2 .

Figure 3 shows the results from the EBS experiments (using the setup seen in 1 right) with Air, argon and nitrogen. As expected, in the argon and nitrogen cases only one peak is present and aligned perfectly with their respective counter parts in the air experiment. 


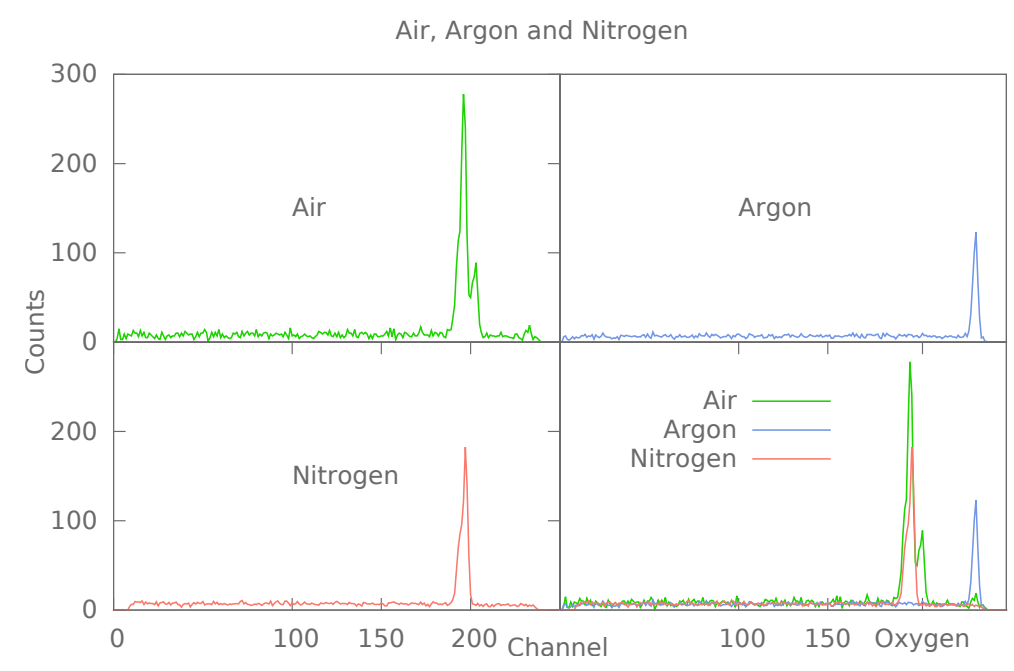

Figure 3: Air, argon and nitrogen EBS, counts vs channel plot.

Additionally, we notice the oxygen peak in the air experiment. The data was subjected to SIMNRA [7] analysis which in turn allowed us to measure the air elemental abundance and the density of the jet.

\section{Ackowlegements}

Special thanks to Professor Dan Shapira from Oak Ridge National Laboratory for his continuous advice and support in the development of this project. To Catalina Stern and Yadira Salazar for the help in the fluid experiment. Also Arcadio Huerta and IFUNAM's shop. This work was done with the support of CONACYT under contracts 51600, 82692, 123655 and DGAPAUNAM IN-118310, IN-112609, IN-105510, IN-115213, TA-100213, IN-103312.

\section{References}

[1] Shapira D, Jr J F, Novotny R, Shivakumar B, Parks R and Thornton S 1985 Nucl. Instr. and Meth. in Physics Research Section A: Accelerators, Spectrometers, Detectors and Associated Equipment 228 259 - 266 ISSN 0168-9002 URL

http://www.sciencedirect.com/science/article/pii/0168900285902670

[2] Becker H, Buchmann L, Gorres J, Kettner K, Krawinkel H, Rolfs C, Schmalbrock P, Trautvetter H and Vlieks A 1982 Nucl. Instr. and Meth. in Phys. Res. 198 277-292 ISSN 0167-5087 URL http://www.sciencedirect.com/science/article/pii/0167508782902654

[3] Kontos A, Schurmann D, Akers C, Couder M, Gorres J, Robertson D, Stech E, Talwar R and Wiescher M 2012 Nucl. Instr. and Meth. in Phys. Res. S. A 664272 - 281 ISSN 0168-9002 URL http://www.sciencedirect.com/science/article/pii/s0168900211019899

[4] Lemut A, Bemmerer D, Confortola F, Bonetti R, Broggini C, Corvisiero P, Costantini H, Cruz J, Formicola A, Fulop Z, Gervino G, Guglielmetti A, Gustavino C, Gyurky G, Imbriani G, Jesus A, Junker M, Limata B, Menegazzo R, Prati P, Roca V, Rogalla D, Rolfs C, Romano M, Alvarez C R, Schumann F, Somorjai E, Straniero O, Strieder F, Terrasi F and Trautvetter H 2006 Phys. Lett. B 634 
483 - 487 ISSN 0370-2693 URL

http://www.sciencedirect.com/science/article/pii/s0370269306001973

[5] Settles G S 2001 Schlieren and shadowgraph techniques (Springer)

[6] Merzkirch W 1987 Flow Visualization (Academic Press) ISBN 9780124913516 URL http: //books.google.com.mx/books?id=u-cgBvCnFf8C

[7] Mayer M 1999 SIMNRA, a simulation program for the analysis of NRA, RBS and ERDA American Institute of Physics Conference Series (American Institute of Physics Conference Series vol 475) pp 541-544 